\title{
A Plea to Reconsider the Diagnosis
}

\author{
Joseph A McBride, MD ${ }^{1,2 *}$, Bryce Binstadt, MD, PhD³, Raheel Ahmed, MD, PhD4, \\ Mustafa K Baskaya, MD', Andrew PJ Olson, MD, Ellen Wald, MD²
}

\begin{abstract}
${ }^{1}$ Department of Medicine, University of Wisconsin-Madison School of Medicine and Public Health, Madison, Wisconsin; ${ }^{2}$ Department of Pediatrics, University of Wisconsin-Madison School of Medicine and Public Health, Madison, Wisconsin; ${ }^{3}$ Department of Pediatrics, University of Minnesota, Minneapolis, Minnesota; ${ }^{4}$ Department of Neurosurgery, University of Wisconsin-Madison School of Medicine and Public Health, Madison, Wisconsin; ${ }^{5}$ Department of Medicine, University of Minnesota, Minneapolis, Minnesota.
\end{abstract}

An eight-month-old unvaccinated boy presented to an emergency department (ED) with fever, neck pain, and lethargy. Examination of the cerebrospinal fluid (CSF) demonstrated hazy fluid with a white blood cell count of 3,906 cells/ uL $(90 \%$ polymorphonuclear cells, $6 \%$ lymphocytes, and $4 \%$ monocytes), 0 red blood cells/uL, protein of $40 \mathrm{mg} / \mathrm{dL}$, and glucose of $56 \mathrm{mg} / \mathrm{dL}$. No organisms were seen on Gram stain. Ceftriaxone and vancomycin were administered. CSF, blood, and urine cultures remained sterile; arbovirus serology was nonreactive, and polymerase chain reactions (PCRs) for enterovirus, Herpes simplex virus (HSV), Streptococcus pneumoniae, Neisseria meningitidis, and Haemophilus influenza were negative. His irritability improved, but his fevers continued. The antibiotics were stopped after 10 days of empiric treatment, and his fever resolved within 36 hours of cessation of antibiotics. He was diagnosed with aseptic meningitis and possible drug fever, attributed to either ceftriaxone or vancomycin.

There are many possibilities to consider in an unimmunized child with signs and symptoms of meningitis. The vaccine-preventable infections are ruled out in the setting of negative cultures and PCRs. While the most common etiology of aseptic meningitis is secondary to viral infections, the considerations of drug fever and aseptic meningitis deserve more attention. A thorough medication history should be taken as nonsteroidal anti-inflammatory drugs (NSAIDs) are relatively commonly linked to aseptic meningitis. Evaluation should focus on family history, medications, and exposures.

There was no family history of meningitis nor known exposures to mosquitos or ticks. The patient did not have a history of atypical or severe infections. He had one episode of acute otitis media that resolved without antibiotics. He had a history of delayed speech and was more irritable than his siblings.

*Corresponding Author: Joseph A. McBride, MD; E-mail: jmcbride@medicine. wisc.edu; Telephone 608-263-8431.

Published online first December 18, 2019

Received: March 15, 2019; Revised: October 24, 2019;

Accepted: October 29, 2019

๑ 2020 Society of Hospital Medicine DOI 10.12788/jhm.3348
Sixteen months later, at two years of age, he presented to his primary care physician in Wisconsin for evaluation of one day of fever and fussiness without rhinorrhea or cough. Examination showed enlarged tonsils without exudate or pharyngeal erythema. His tympanic membranes were normal, and the lung fields were clear. Two of his older siblings and his father had been diagnosed with streptococcal pharyngitis and were receiving antibiotic treatment. A rapid streptococcal antigen test was positive, and amoxicillin started.

Group A streptococcal (GAS) pharyngitis is an acute infection of the oropharynx or nasopharynx caused by Streptococcus pyogenes and is most common in school-aged children. GAS pharyngitis is less common at age two years unless there is definite exposure. The most frequent presentations in this age group ( $<3$ years of age) include protracted nasal symptoms (congestion and rhinorrhea) and cough instead of a well-localized episode of pharyngitis.

The amoxicillin was continued for four days without improvement in fever or fussiness. His oral intake decreased, and he developed nonbilious, nonbloody emesis without diarrhea. He followed up with his pediatrician for the presumed streptococcal pharyngitis. Because of the previous concern for drug fever related to his ceftriaxone exposure, the amoxicillin was discontinued. Supportive care was recommended.

While viral infections remain the most likely etiology, noninfectious etiologies, such as vasculitis, should be considered. Kawasaki disease should be considered in any child with prolonged fever. Kawasaki disease can also cause aseptic meningitis that could provide an explanation for his original episode at eight months of age; nevertheless, it is rare for Kawasaki disease to recur.

Over the next three days, his temperature was as high as $38.8^{\circ} \mathrm{C}\left(101.8^{\circ} \mathrm{F}\right)$, he became more irritable, and his vomiting worsened; his family believed he had a headache. He was again seen by his pediatrician, now with eight days of fever. On examination, his oropharynx was mildly erythematous with palatal petechiae and $2+$ tonsillar enlargement; shotty anterior cervical lymphadenopathy was present. Concern for incompletely treated streptococcal pharyngitis prompted prescription of azithromycin for five days. 
This information does not change the differential diagnosis significantly. Azithromycin is as effective as beta lactams for the treatment of GAS pharyngitis if the GAS is susceptible to macrolides. Macrolide resistance rates vary between communities and have been as high as $15 \%$ in Wisconsin; knowledge of local resistance patterns is important. ${ }^{1}$

Despite the azithromycin, his symptoms worsened, and he became lethargic. The family believed the symptoms were similar to those during his previous episode of meningitis. They presented to an ED where he was febrile to $39.4^{\circ} \mathrm{C}$ $\left(102.9^{\circ} \mathrm{F}\right)$ with a heart rate of 159 beats per minute and blood pressure of $113 / 84 \mathrm{~mm} \mathrm{Hg}$. His head circumference was 50.5 $\mathrm{cm}$ (97th percentile) compared with his weight of $10.8 \mathrm{~kg}$ (23.81 lbs; 22nd percentile). He was listless when undisturbed and irritable during the examination; his neck was supple and strong, and reflexes were normal. The remainder of his examination, including joints and skin, was normal. His white blood cell count was $18.6 \mathrm{~K} / \mathrm{uL}$, hemoglobin $11.8 \mathrm{~g} / \mathrm{dL}$, and platelets $401 \mathrm{~K} / \mathrm{uL}$. A chest radiograph was normal.

The patient is presenting on the 10th day of fever-a long time for any patient to remain febrile. Although most typically due to infectious etiologies, rheumatologic and oncologic diseases must be considered. It is important to characterize the pattern of fevers during the past 10 days and whether the patient has had similar febrile illnesses in the past. In this case, his past medical history substantially alters the differential diagnosis. The positive rapid strep test and history of recent strep pharyngitis are of uncertain importance, and the patient's nonresponsiveness to antibiotics should raise concern for a second disease process (other than streptococcal infection) causing the fever. His unimmunized status changes the pretest probability of serious conditions such as bacterial meningitis caused by $S$. pneumoniae. A lumbar puncture should be performed, including an opening pressure; if the CSF again shows pleocytosis, but no infectious etiology is identified, then imaging of the brain (magnetic resonance imaging [MRI] or magnetic resonance angiogram) should be performed to evaluate for anatomic abnormalities.

CSF examination revealed 9,327 white blood cells/uL (82\% polymorphonuclear cells, $1 \%$ lymphocytes, and $17 \%$ monocytes), 114 red blood cells/uL, protein of $87 \mathrm{mg} /$ $\mathrm{dL}$, and glucose of $63 \mathrm{mg} / \mathrm{dL}$. Gram stain revealed no organisms. Ceftriaxone, vancomycin, and acyclovir were started, and he was transferred to a children's hospital.

This history must be viewed through two alternate lenses: that the two episodes of meningitis are related or that they are unrelated. The finding of a neutrophil predominance in the CSF in the setting of aseptic (or nonbacterial) meningitis is less common than a lymphocytic predominance. Most commonly, aseptic meningitis is due to viral infection and is typically associated with a lymphocyte predominance, although a moderate neutrophil predominance can be seen in patients with enterovirus meningitis. Neutrophil-predominant aseptic meningitis can also accompany genetic auto-inflammatory syndromes (eg, familial Mediterranean fever and cryopyrin-associated periodic syndrome). This finding can also be seen in other noninfectious conditions such as neurosarcoidosis, Behçet's disease, Cogan syndrome, and other vasculitides. Drug-induced aseptic meningitis can also cause neutrophil predominance. Additionally, the apparent neutrophil predominance could be explained if the patient had lymphopenia associated with primary or acquired immunodeficiency; therefore, the peripheral leukocyte differential obtained at the same time as the CSF should be evaluated. However, immunodeficiency is less likely given the patient's lack of history of recurrent infections.

The main objective information added here is that the patient now has his second episode of likely aseptic meningitis with neutrophilic predominance, although it is possible that antibiotic therapy may have led to a false-negative CSF culture. However, this possible partial treatment was not a consideration in the first episode of meningitis. Having two similar episodes increases the likelihood that the patient has an underlying inflammatory/immune disorder, likely genetic (now termed "inborn errors of immunity"), or that there is a common exposure not yet revealed in the history (eg, drug-induced meningitis). Primary immunodeficiency is less likely than an autoinflammatory disease, considering the patient's course of recovery with the first episode.

Further evaluation of the CSF did not reveal a pathogen. Bacterial CSF culture was sterile, and PCRs for HSV and enterovirus were negative.

The differential diagnosis is narrowing to include causes of recurrent, aseptic, neutrophilic meningitis. The incongruous head circumference and weight could be due to a relatively large head, a relatively low weight, or both. To interpret these data properly, one also needs to know the patient's length, the trajectory of his growth parameters over time, and the parents' heights and head circumferences. One possible scenario, considering the rest of the history, is that the patient has a chronic inflammatory condition of the central nervous system (CNS), leading to hydrocephalus and macrocephaly. It is possible that systemic inflammation could also lead to poor weight gain.

When considering chronic causes of aseptic meningitis associated with neutrophil predominance in the CSF, autoinflammatory disorders (cryopyrin-associated periodic syndrome, Muckle-Wells syndrome, neonatal-onset multisystem inflammatory disease [NOMID], and chronic infantile neurological cutaneous articular syndrome [CINCA]) should be considered. The patient lacks the typical deforming arthropathy of the most severe NOMID/CINCA phenotype. If the brain imaging does not reveal another etiology, then genetic testing of the patient is indicated.

Because of the history of recurrent meningitis with marked neutrophilic pleocytosis, yet no evidence of infection given normal glucose, only mildly elevated protein, and no culture growth, an MRI of the brain was obtained. 


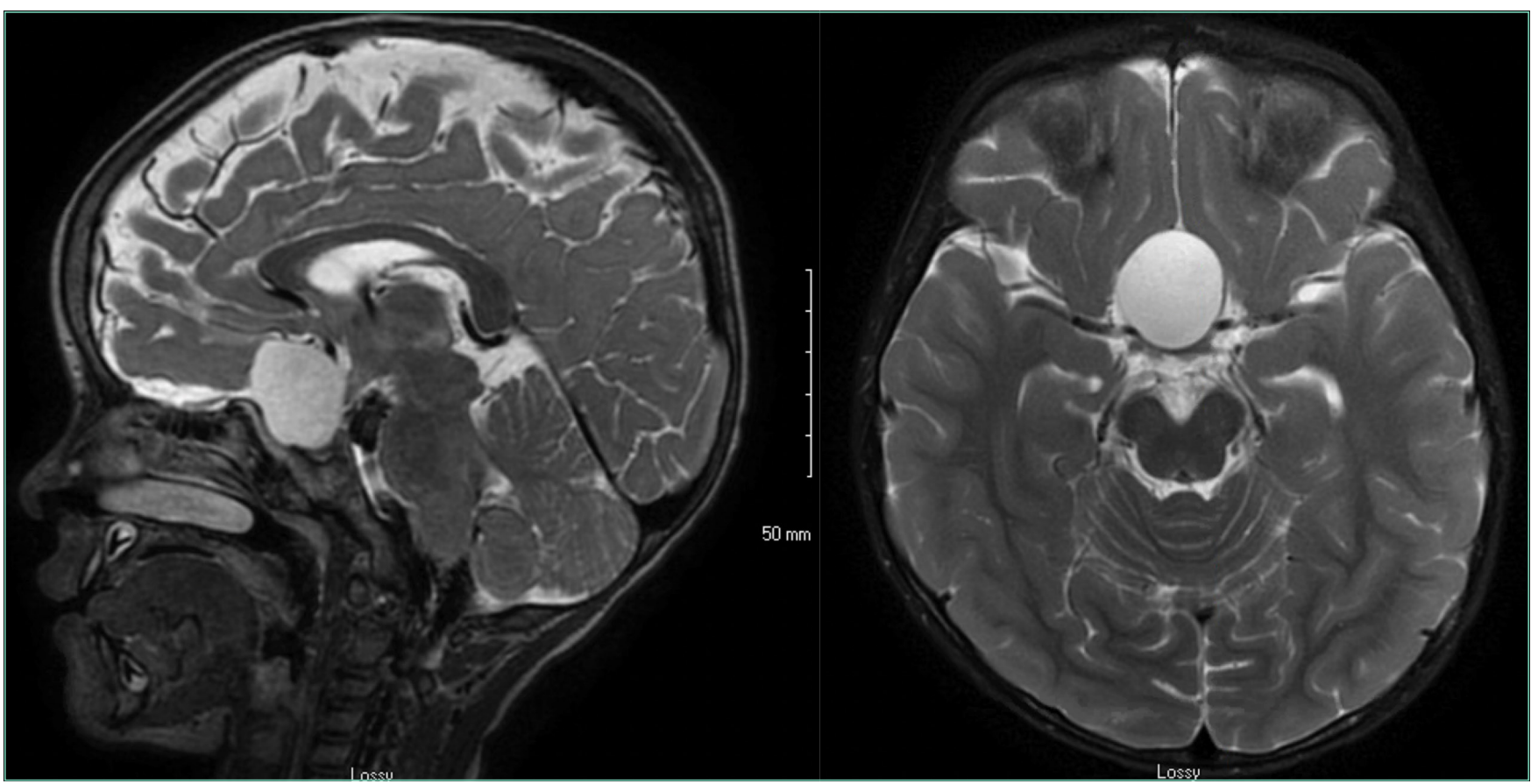

FIG. Magnetic Resonance Image of the Brain. A $2.6 \mathrm{~cm}$ suprasellar cystic mass with a thin rim of enhancement is seen.

MRI revealed a sharply circumscribed, homogeneous, nonenhancing $2.6 \mathrm{~cm}$ diameter cystic suprasellar mass with a thin rim of capsular enhancement (Figure). The appearance was most consistent with an epidermoid cyst, a dermoid, Rathke's cleft cyst (RCC), or, less likely, a craniopharyngioma. The recurrent aseptic meningitis was attributed to chemical meningitis secondary to episodic discharging of the tumor. There was no hydrocephalus on imaging, and the enlarged head circumference was attributed to large parental head circumference.

Antibiotics were discontinued and supportive care continued. A CSF cholesterol level of $4 \mathrm{mg} / \mathrm{dL}$ was found (normal range $0.6 \pm 0.2 \mathrm{mg} / \mathrm{dL}$ ) on the CSF from admission. Fevers and symptoms ultimately improved with 72 hours of admission. He was discharged with neurosurgical follow-up, and within a year, he developed a third episode of aseptic meningitis. He eventually underwent a craniotomy with near-total resection of the cyst. Histopathological analysis indicated the presence of an underlying RCC, despite initial clinical and radiographic suspicion of an epidermoid cyst. He recovered well with follow-up imaging demonstrating stable resolution of the RCC and no further incidents of aseptic meningitis in the 12 months since the surgery.

\section{DISCUSSION}

Aseptic meningitis is defined as meningitis with negative bacterial cultures from CSF and is habitually equated with viral meningitis. ${ }^{2}$ This erroneous equivalence may curb critical thinking about alternative diagnoses, as aseptic meningitis may also be associated with a wide range of both infectious and noninfectious etiologies (Table). A thorough history and physical examination are the essential first steps in determining the etiology of aseptic meningitis, as many of the listed etiologies can be effectively eliminated through the evaluation of risk factors and exposures. Laboratory evaluation of CSF including cell count with differential, glucose, and protein levels is required. Gram stain and culture should be obtained to evaluate for bacterial meningitis even if suspicion is low. Multiplex and dedicated PCRs to viral agents as well as a serologic test for arboviruses, are widely available. Multiple episodes of aseptic meningitis with HSV, known as Mollaret's meningitis, or enterovirus, which is more common in males with X-linked agammaglobulinemia, should be considered in patients with recurrent disease. Imaging is not indicated for every patient with aseptic meningitis; however, if anatomic abnormalities or malignancy are suspected, or in the evaluation of recurrent disease, then an MRI of the brain should be considered.

This case highlights how the analysis of CSF pleocytosis is not always predictive of a specific underlying etiology. The classic teaching that viral meningitis is associated with lymphocytic pleocytosis is based on studies of mumps meningitis. ${ }^{3}$ It is important to recognize that a neutrophilic pleocytosis is also described in viral infections including those caused by an enterovirus, herpes simplex, and arboviruses. ${ }^{4,5}$ Moreover, while the magnitude of the neutrophilic pleocytosis should always raise suspicion of bacterial meningitis, it should also be associated with hypoglycorrhachia and elevated CSF protein levels. Antibiotic pretreatment of bacterial meningitis can alter CSF chemistries (raise CSF glucose levels and lower CSF protein levels), but chemistries are unlikely to return completely to normal. ${ }^{6}$ In this case, one clue that hinted toward a noninfectious etiology was the recurrence of relatively normal CSF glucose and protein levels in the setting of such a highly inflammatory pleocytosis on multiple occasions. 


\section{TABLE. Infectious and Noninfectious Etiologies of Aseptic Meningitis}

\begin{tabular}{ll}
\hline Infectious & Noninfectious \\
\hline Bacteria: & Drugs/Blood Products: \\
Bacterial endocarditis & Allopurinol \\
Bartonellosis & Anti-CD3 monoclonal antibody \\
Brain abscess & Azathioprine \\
Brucellosis & Beta lactams (Amoxicillin) \\
Ehrlichiosis & Carbamazepine \\
Leptospirosis & Intrathecal drugs \\
Lyme disease & Intravenous immunoglobulin \\
Mycoplasma pneumoniae & Isoniazid \\
Nocardiosis & Nonsteroidal anti-inflammatory drugs (NSAIDs) \\
Parameningeal infection & Trimethoprim-sulfamethoxazole \\
Partially treated bacterial meningitis & \\
Rocky Mountain Spotted Fever & \\
Syphilis & \\
Typhus &
\end{tabular}

\section{Fungi:}

Blastomycosis

Cryptococcosis

Coccidioidomycosis

Histoplasmosis

\section{Inflammatory:}

Autoimmune encephalitis

Behçet's disease

CNS vasculitis

Granulomatosis with polyarteritis

Kawasaki's disease

Multiple sclerosis

Rheumatoid arthritis

Sarcoidosis

Systemic lupus erythematosus

Mediterranean Fever

Cryopyrin-associated periodic syndrome (CAPS)

Cogan syndrome

Muckle-Wells syndrome

Neonatal-onset multisystem inflammatory disease (NOMID)

Chronic infantile neurological cutaneous articular syndrome (CINCA)

\section{Mycobacterial:}

Tuberculosis

Disseminated Mycobacterium avium complex

\section{Neoplastic:}

Carcinomatous meningitis

CNS Lymphoma

Leukemia

Posttransplantation lymphoproliferative disorder

\section{Parasites:}

Angiostrongylus cantonensis

Neurocystericercosis

Primary amoebic meningoencephalitis (Naeglaria)

Toxoplasmosis

Trinchinosis

\section{Postinfectious/Vaccine Related:}

Measles

Pertussis vaccine

Rabies vaccine

Rubella

Smallpox vaccine

Varicella

Variola

Yellow fever vaccine

\section{Miscellaneous:}

Chemical meningitis

Epidermoid cysts

Rathke's cleft cysts

Migraines

Postsurgical

HIV

Influenza A \& B

Lymphocytic choriomeningitis

Measles

Mumps

Abbreviations: CMV, Cytomegalovirus; EBV, Epstein-Barr virus; HHV, Human Herpesvirus; HIV, human immunodeficiency virus; HSV, Herpes simplex viruses; VZV, varicella-zoster virus 
There is a wide spectrum of CNS mass lesions known for causing chemical meningitis including epidermoid, dermoid, craniopharyngiomas, and RCCs. While imaging can be suggestive, histological examination is often required to make a specific diagnosis. In this patient, the diagnosis of chemical meningitis secondary to a ruptured brain tumor was confirmed by MRI. CNS tumors that may cause aseptic meningitis are typically slow-growing lesions that cause symptoms due both to local growth and regional neurovascular compression. These masses can rupture and disseminate inflammatory contents into the subarachnoid space giving rise to chemical aseptic meningitis. Their contents may include materials rich in keratin, cholesterol, and lipids, which cause an intense sterile inflammatory reaction when discharged, possibly via cholesterol activation of the inflammasome. ${ }^{7,8}$ The subsequent inflammatory response produces a neutrophilic pleocytosis, often suggestive of bacterial meningitis, while simultaneously maintaining the near normalcy of the CSF glucose and protein levels. Elevated levels of CSF cholesterol may raise suspicion of the diagnosis. Not all discharging tumors result in purely chemical meningitis, as secondary bacterial meningitis with $S$. pneumoniae or other respiratory flora can coexist if cysts communicate with the respiratory tract. ${ }^{9}$

Rathke's cleft is formed during the development of the pituitary gland by the evagination of oral ectoderm through the precursor of the oral cavity. ${ }^{10}$ The cleft gives rise to the endocrine cells of the anterior pituitary. It subsequently disconnects from the oral cavity and develops into the pars intermedia between the anterior and posterior pituitary. Cystic enlargement of Rathke's cleft through epithelial proliferation and secondary secretions leads to the development of an RCC. RCCs are nonneoplastic lesions, and the majority are diagnosed incidentally. Asymptomatic RCCs are common and are detected in 13\%$22 \%$ of routine autopsies. ${ }^{11}$ Symptomatic lesions may present with headaches due to mechanical effects on pain-sensitive dura or cranial nerves. Severe acute onset headaches may arise secondary to pituitary hemorrhage. RCCs can also cause ophthalmic or endocrinological impairment due to sellar compression. As in the present case, rarely cystic rupture and subarachnoid extravasation of epithelial-derived contents lead to a chemical aseptic meningitis. ${ }^{12}$

Surgical resection is indicated for symptomatic RCCs that exert neurologic, ophthalmic, or endocrinological symptoms. ${ }^{13}$ The surgical goal is the removal of the lesion and complete excision of the capsule unless it is extremely adherent to neurovascular structures. Surgical morbidity is related to the risk of hypopituitarism, visual decline, incomplete resection with lesion regrowth, and aseptic meningitis. Surgical approaches to this region are potentially complicated by proximity to optic nerves, pituitary glands, major arteries, and perforating vessels belonging to the circle of Willis. In addition, potential dehiscence of the skull base floor due to progressive cyst growth can give rise to a delayed risk of CSF leak and complicate surgical recovery. Surgery was indicated for this patient because of the parasellar location of his cyst placing him at risk for visual decline due to compression of the optic chiasm as well as pituitary dysfunction or obstructive hydrocephalus from ventricular compression.

This case is illustrative for learning because, at the outset, there were many possibilities to explore in an unimmunized child with meningitis. This patient's neutrophilic cell count and partial antibiotic treatment only compounded the certainty of a bacterial etiology. However, further scrutiny of the history and laboratory parameters revealed the true underlying diagnosis of RCC. Ultimately, the plea to reconsider the pleocytosis was heard.

\section{KEY LEARNING POINTS}

- The CSF cell count and differential should be used in conjunction with CSF chemistries (glucose and protein) to raise or lower suspicion of bacterial meningitis.

- Aseptic meningitis is a syndrome and not a specific diagnosis. Clinicians should be alert to key aspects of the history and physical examination, which prompt consideration of noninfectious etiologies.

- Aseptic chemical meningitis secondary to discharging CNS tumors, including RCCs, should be considered in episodes of recurrent culture-negative meningitis.

Disclosures: The authors have no financial conflicts of interest or disclosures.

\section{References}

1. DeMuri GP, Sterkel AK, Kubica PA, Duster MN, Reed KD, Wald ER. Macrolide and clindamycin resistance in group a streptococci isolated from children with pharyngitis. Pediatr Infect Dis J. 2017;36(3):342-344. https://doi. org/10.1097/INF.0000000000001442.

2. Lee BE, Davies HD. Aseptic meningitis. Curr Opin Infect Dis. 2007;20(3):272277. https://doi.org/10.1097/QCO.0b013e3280ad4672.

3. Ritter BS. Mumps meningoencephalitis in children. J Pediatr. 1958;52(4):424433. https://doi.org/10.1016/S0022-3476(58)80063-3.

4. Miller SA, Wald ER, Bergman I, DeBiasio R. Enteroviral meningitis in January with marked cerebrospinal fluid pleocytosis. Pediatr Infect Dis. 1986;5(6):706707. https://doi.org/10.1097/00006454-198611000-00024.

5. Jaijakul S, Salazar L, Wootton SH, Aguilera E, Hasbun R. The clinical significance of neutrophilic pleocytosis in cerebrospinal fluid in patients with viral central nervous system infections. Int J Infect Dis. 2017;59:77-81. https://doi. org/10.1016/j.ijid.2017.04.010

6. Nigrovic LE, Malley R, Macias CG, et al. Effect of antibiotic pretreatment on cerebrospinal fluid profiles of children with bacterial meningitis. Pediatrics. 2008;122(4):726-730. https://doi.org/10.1542/peds.2007-3275.

7. Cherian A, Baheti NN, Easwar HV, Nair DS, lype T. Recurrent meningitis due to epidermoid. J Pediatr Neurosci. 2012;7(1):47-48. https://doi. org/10.4103/1817-1745.97624

8. Grebe A, Latz E. Cholesterol crystals and inflammation. Curr Rheumatol Rep. 2013;15(3):313. https://doi.org/10.1007/s11926-012-0313-z.

9. Kriss TC, Kriss VM, Warf BC. Recurrent meningitis: the search for the dermoid or epidermoid tumor. Pediatr Infect Dis J. 1995;14(8):697-700.

10. Bresson D, Herman P, Polivka M, Froelich S. Sellar lesions/pathology. Otolaryngol Clin North Am. 2016;49(1):63-93. https://doi.org/10.1016/j. otc.2015.09.004

11. Billeci D, Marton E, Tripodi M, Orvieto E, Longatti P. Symptomatic Rathke's cleft cysts: a radiological, surgical and pathological review. Pituitary. 2004;7(3):131-137. https://doi.org/10.1007/s11102-005-1755-3.

12. Steinberg GK, Koenig GH, Golden JB. Symptomatic Rathke's cleft cysts. Report of two cases. J Neurosurg. 1982;56(2):290-295. https://doi.org/10.3171/ jns.1982.56.2.0290.

13. Zada G. Rathke cleft cysts: a review of clinical and surgical management. Neurosurg Focus. 2011;31(1):E1. https://doi.org/10.3171/2011.5.FOCUS1183. 